Published in final edited form as:

Radiother Oncol2013 June ; 107(3): 372-376. doi:10.1016/j.radonc.2013.05.001.

\title{
Genome-wide association study identifies a region on chromosome 11q14.3 associated with late rectal bleeding following radiation therapy for prostate cancer
}

\author{
Sarah L. Kerns, Ph.D., M.P.H. ${ }^{1,2}$, Richard Stock, M.D. ${ }^{1}$, Nelson N. Stone, M.D. ${ }^{1,3}$, Seth R. \\ Blacksburg, M.D., M.B.A. ${ }^{1}$, Lynda Rath, B.F.A. ${ }^{1}$, Ana Vega, Ph.D. ${ }^{4}$, Laura Fachal, M.Sc. ${ }^{4}$, \\ Antonio Gómez-Caamaño, M.D. ${ }^{5}$, Dirk De Ruysscher, M.D., Ph.D. ${ }^{6,7}$, Guido Lammering, \\ M.D., Ph.D. ${ }^{7}$, Matthew Parliament, M.D. ${ }^{8}$, Michael Blackshaw, M.D. ${ }^{8}$, Michael Sia, M.D. ${ }^{9}$, \\ Jamie Cesaretti, M.D. ${ }^{10}$, Mitchell Terk, M.D. ${ }^{10}$, Rosetta Hixson, M.D. ${ }^{10}$, Barry S. Rosenstein,

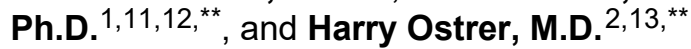

1Department of Radiation Oncology, Mount Sinai School of Medicine, New York, NY ${ }^{2}$ Department of Pathology, Albert Einstein College of Medicine, Bronx, NY ${ }^{3}$ Department of Urology, Mount Sinai School of Medicine, New York, NY ${ }^{4}$ Fundación Pública Galega de Medicina XenómicaSERGAS, Grupo de Medicina Xenómica-USC, CIBERER, IDIS, Santiago de Compostela, Spain ${ }^{5}$ Department of Radiation Oncology, Complexo Hospitalario Universitario de Santiago, SERGAS, Santiago de Compostela, Spain ${ }^{6}$ Department of Radiation Oncology, University Hospital Leuven, $\mathrm{KU}$ Leuven, Belgium ${ }^{7}$ Department of Radiation Oncology (Maastro clinic/GROW), Maastricht University Medical Center, Maastricht, The Netherlands ${ }^{8}$ Division of Radiation Oncology, Department of Oncology, Cross Cancer Institute, University of Alberta, Edmonton, Canada ${ }^{9}$ Department of Radiation Oncology, Tom Baker Cancer Center, University of Calgary, Calgary, Canada ${ }^{10}$ Florida Radiation Oncology Group, Jacksonville, FL, United States ${ }^{11}$ Department of Radiation Oncology, New York University School of Medicine, New York, NY ${ }^{12}$ Departments of Dermatology and Preventive Medicine, Mount Sinai School of Medicine, New York, NY

${ }^{13}$ Department of Genetics, Albert Einstein College of Medicine, Bronx, NY

\section{Abstract}

Background and Purpose-Rectal bleeding can occur following radiotherapy for prostate cancer and negatively impacts quality of life for cancer survivors. Treatment and clinical factors do not fully predict for rectal bleeding, and genetic factors may be important.

Materials and Methods-A genome-wide association study (GWAS) was performed to identify SNPs associated with development of late rectal bleeding following radiotherapy for

\footnotetext{
* Collaboration developed under the framework of the Radiogenomics Consortium

(C) 2013 Elsevier Ireland Ltd. All rights reserved.

Corresponding author: Barry S. Rosenstein, Ph.D., Department of Radiation Oncology, Box 1236, Mount Sinai School of Medicine, One Gustave L. Levy Place, New York, NY 10029. Tel: 212-241-9408, Fax: 212-996-8927, barry.rosenstein@mssm.edu. Equal contributions as senior authors to this manuscript

Conflict of Interest

N.N. Stone is a consultant for Amgen, Ferring, Janssen, Diversified Conference Management, Prologics LLC, and Nihon MediPhysics. R.G. Stock has received payment for lectures and preparation of educational materials from Bard. All other authors report no conflicts of interest.

Publisher's Disclaimer: This is a PDF file of an unedited manuscript that has been accepted for publication. As a service to our customers we are providing this early version of the manuscript. The manuscript will undergo copyediting, typesetting, and review of the resulting proof before it is published in its final citable form. Please note that during the production process errors may be discovered which could affect the content, and all legal disclaimers that apply to the journal pertain.
} 
prostate cancer. Logistic regression was used to test association between 614,453 SNPs and rectal bleeding in a discovery cohort (79 cases, 289 controls), and top-ranking SNPs were tested in a replication cohort (108 cases, 673 controls) from four independent sites.

Results_rs7120482 and rs17630638, which tag a single locus on chromosome 11q14.3, reached genome-wide significance for association with rectal bleeding (combined p-values $5.4 \times 10^{-8}$ and $6.9 \times 10^{-7}$ respectively). Several other SNPs had p-values trending towards genome-wide significance, and a polygenic risk score including these SNPs shows a strong rank-correlation with rectal bleeding (Sommers' $\mathrm{d}=5.0 \times 10^{-12}$ in the replication cohort).

Conclusions-This GWAS identified novel genetic markers of rectal bleeding following prostate radiotherapy. These findings could lead to development of a predictive assay to identify patients at risk for this adverse treatment outcome so that dose or treatment modality could be modified.

\section{Keywords}

radiogenomics; prostate cancer; rectal toxicity; genome-wide association study

\section{Introduction}

Prostate cancer is one of the most common cancers in the world, with approximately one million new cases diagnosed per year worldwide [1]. Prostate cancer can be successfully treated when detected early, by radiotherapy (brachytherapy or intensity-modulated radiation therapy (IMRT)) and/or surgery, and risk of side effects becomes a deciding factor when choosing among treatment options [2]. Even with improvements in precision of radiation delivery, some men experience late adverse effects of radiotherapy for prostate cancer, including rectal bleeding. The incidence of rectal bleeding is dependent, in part, on dosimetry or underlying medical conditions. For example, a multivariate analysis of clinical and patient-specific factors among approximately 780 men treated under the UK Medical Research Council RT01 trial found that prescribed dose and age were significantly associated with risk of rectal toxicity [3]. But, variability in dose and demographic factors does not fully explain the variability in rectal bleeding, and prior studies suggest there are underlying genetic risk factors [4-5].

Previous studies have investigated associations between SNPs in candidate genes, mainly genes involved in DNA damage response and inflammation, and rectal toxicity in prostate cancer patients treated with radiotherapy [6]. However, most of these SNPs have either been investigated in only one study or have shown conflicting results across studies. Few, if any, of these associations have been replicated. A large validation study was recently published in which 92 SNPs in 46 genes were investigated in 637 prostate radiotherapy patients [7]. Five rectal endpoints (bleeding, proctitis, sphincter control, stool frequency, tenesmus) were evaluated in patients two years after radiotherapy. None of the previously reported associations for candidate gene SNPs could be confirmed in this project.

In order to take a broader, unbiased approach, we carried out a two-stage GWAS to identify SNPs associated with late rectal bleeding following radiation therapy for prostate cancer.

\section{Materials and Methods}

\section{Patient Characteristics}

The study included men treated with definitive radiation for biopsy proven adenocarcinoma of the prostate (low, intermediate and high risk). Patients were recruited from the Mount Sinai Medical Center in the USA (MSMC), the Clinical University Hospital of Santiago de 
Compostela in Spain (CHUS), the Maastricht Radiation Oncology Clinic in the Netherlands (MAASTRO), the Cross Cancer Institute and Tom Baker Cancer Centre in Canada (CCI), and the Florida Radiation Oncology Group in the USA (FROG). The MSMC cohort served as a discovery set and the pooled CHUS, MAASTRO, CCI and FROG cohorts served as a replication set. The study was approved by the Institutional Review Boards of the respective study sites, and all men provided informed consent.

The MSMC discovery cohort included 764 patients, of which 368 were included in the GWAS (all cases and a randomly selected set of 289 controls). Unselected controls were similar to selected controls with respect to clinical and demographic variables (Table 1). Treatment included brachytherapy (206; 56\%), brachytherapy with external beam radiation therapy (EBRT) (160; 43.5\%), or EBRT alone (2, 0.5\%). ${ }^{125}$ I (160Gy; TG-43) was used in patients who received brachytherapy alone and ${ }^{103} \mathrm{Pd}(124 \mathrm{~Gy})$ in patients who also received EBRT. The EBRT prescription dose was 45Gy in 25 fractions. All implants were performed using a real time ultrasound guided technique previously described [8-9]. 194 patients (52.7\%) received hormonal therapy in addition to radiotherapy.

In the pooled replication set, the CHUS cohort consisted of 403 patients from the RADIOGEN trial treated with EBRT [10]. Total delivered dose ranged from 50 to 76 (median 74) Gy with 1.8 to 2.0 Gy per fraction. 311 (77.2\%) patients also received hormonal therapy. The MAASTRO cohort consisted of 203 patients treated with EBRT alone (144 patients; 70.9\%) prescribed to 68-72Gy in 2.0 Gy fractions or with brachytherapy alone (59 patients; 29.1\%) prescribed to 145Gy. 111 patients (54.7\%) also received hormonal therapy. The CCI cohort consisted of 153 patients treated with EBRT. Hypo-fraction regimens were given to $33(21.6 \%)$ patients and consisted of 68Gy in 25 fractions or 55Gy in 16 fractions. Standard fraction was delivered to 120 (78.4\%) patients with 72 to 82Gy delivered in 1.8 to 2Gy per fraction. Hormonal therapy was given to 77 (50.3\%) patients. The FROG cohort consisted of 52 patients. The treatment regimen and technique were similar to the MSMC group. Treatment included ${ }^{125}$ I brachytherapy (5 patients; $9.6 \%$ ), ${ }^{103} \mathrm{Pd}$ brachytherapy with EBRT (38 patients; 73.1\%), or EBRT alone (9 patients; 17.2\%). 28 patients (53.8\%) received hormonal therapy as part of their treatment. EBRT patients were all treated with IMRT technique. Total doses ranged from 64.8 to $81 \mathrm{~Gy}$ using 1.8Gy fractions. Total biologically effective dose was calculated among all cohorts using previously published methods with an / of 2 [11].

All men were followed prospectively for development of rectal bleeding. Included patients had at least one year of follow-up, and late ( $>90$ days post-RT) rectal bleeding was considered. Rectal bleeding was assessed as follows: in the MSMC and FROG patients using the Radiation Therapy Oncology Group (RTOG) late radiation morbidity scoring schema; in the CHUS and CCI patients using National Cancer Institute Common Toxicity Criteria for Adverse Events (CTCAE) version 3; and in the MAASTRO patients using a patient self-report questionnaire, which was harmonized to the RTOG grading scheme (see Appendix for detailed descriptions). For all cohorts, rectal bleeding grade was assigned by a physician based on patient-reported description of symptoms. Care was taken to ensure comparability in case/control definitions across cohorts. Patients with grade 2 or higher rectal bleeding were considered cases and patients with grade 0 or 1 were considered controls.

\section{Genotyping and Quality Control}

Genomic DNA was isolated from lymphocytes. DNA from men in the discovery cohort was genotyped for 900,000 SNPs using Affymetrix v6.0 genome-wide arrays, and genotypes were called using Genotyping Console (Affymetrix, Santa Clara, CA). DNA from men in the CHUS, MAASTRO and FROG replication cohorts was genotyped using Illumina iSelect 
custom SNP arrays, and genotypes were called by GenomeStudio (Illumina, San Diego, CA). DNA from men in the CCI cohort was genotyped using Affymetrix v6.0 genome-wide arrays, but these samples were only analyzed for SNPs included in the replication study and present on the Illumina array. SNPs were excluded from analysis if missing in $>5 \%$ of samples (137,589 SNPs in the discovery dataset; 14 SNPs in the replication dataset), if they had minor allele frequency (MAF) $<5 \%(157,580$ SNPs in the discovery dataset), or if they showed deviation from Hardy-Weinberg equilibrium ( $\mathrm{p}$-value $<1 \times 10^{-5} ; 5,433$ SNPs in the discovery dataset). Individuals were excluded if they showed cryptic relatedness (8 pairs) or if they had call rate $<90 \%$ (24 individuals). Duplicate samples and a trio of HapMap samples were included in both rounds of genotyping and showed $>98 \%$ concordance. PLINKv1.07 was used for quality control and SNP association tests [12].

Principal components analysis (PCA) was performed to assess and control for individual genetic ethnicity using 11 reference populations from the International HapMap Project [13]. PCA was performed using 860 ancestry-informative markers. SNP data processing and PCA was carried out using R [14].

\section{Statistical Analysis}

Association between clinical and demographic variables and rectal bleeding was assessed using chi-square test and one-way analysis of variance. IBM SPSS Statistics 19 was used for statistical tests.

Tests for association between each SNP and rectal bleeding were carried out using multivariable logistic regression including the first five principle components (to control for ethnicity) as well as study site in the replication stage (to control for differences in treatment across the four sites). Fisher's trend method was used to combine p-values from the discovery and replication studies [15].

A polygenic risk score was computed by assigning a numeric count of risk alleles for each SNP and then adding together the number of risk alleles for the replicated SNPs. For SNPs showing an additive inheritance mode, homozygotes for the risk allele were assigned 1 point, heterozygotes were assigned 0.5 points, and homozygotes for the non-risk allele were assigned 0 points. For SNPs with either dominant or recessive inheritance mode, the appropriate genotype categories were collapsed so that those with the risk genotype(s) were assigned 1 point and those without the risk genotype(s) were assigned 0 points. Rankcorrelation between the polygenic risk score and rectal bleeding was assessed using Somers' d.

\section{Results}

Among 1,572 men with prostate adenocarcinoma who met the inclusion criteria for the study, 188 (12.0\%) developed significant ( $\geq$ grade 2 or equivalent severity; Appendix 1) late rectal bleeding. The incidence of rectal bleeding was similar across study sites: 80 out of $764(10.5 \%)$ at MSMC; 41 out of $403(10.2 \%)$ at CHUS; 36 out of $203(17.7 \%)$ at MAASTRO; 26 out of 153 (17.0\%) at CCI; and 6 out of 52 (11.5\%) at FROG. The mean length of follow-up for assessing rectal bleeding was similar between cases and controls in both the discovery and replication cohorts (50 months for cases vs. 48 months for controls in the MSMC cohort and 40 months for cases vs. 41 months for controls in the replication).

A genome-wide association study (GWAS) was performed to identify SNPs associated with development of rectal bleeding in the MSMC discovery cohort (79 cases and 289 controls), and top SNPs were investigated in the pooled replication cohort (108 cases and 673 controls). Table 1 shows the characteristics of the patients included in the GWAS. On 
univariate analysis, there were no statistically significant differences $(\mathrm{p}<0.05)$ in age, Gleason score, treatment modality, or use of hormonal therapy between cases and controls in either the MSMC cohort or the pooled replication cohort. Total biologically effective dose (BED) was similar between cases and controls in the MSMC cohort and the pooled replication cohort, though the majority of the MSMC patients were treated with brachytherapy whereas the replication cohort patients were largely treated with external beam RT and received, on average, lower doses (Table 1).

After quality control, 614,453 SNPs were investigated for association with rectal bleeding in the MSMC discovery cohort. Consistent with self-reported race/ethnicity, PCA confirmed that the majority of the patients were of European ancestry, with approximately $25 \%$ showing African, Hispanic, and/or Asian ancestry (Supplementary Figure 1). Inclusion of the first five principle components in association tests adequately controlled for ethnicity as evidenced by a low genomic inflation factor (1.02) after correction (Supplementary Figure 2). A total of 491 SNPs were identified with $\mathrm{p}$-values between $3.1 \times 10^{-10}$ and $9.9 \times 10^{-4}$ in the discovery cohort, and these SNPs were tested in the pooled replication cohort. A region on chromosome 11q14.3 contained two SNPs, rs7120482 and rs17630638, which have Fisher combined p-values reaching or near genome-wide significance $\left(5.4 \times 10^{-8}\right.$ and $6.9 \times 10^{-7}$ respectively) and show the same odds ratio directionality and inheritance model in the discovery and replication cohorts (Supplementary Table 1). These two SNPs are in strong linkage disequilibrium $\left(\mathrm{r}^{2}=1.0\right.$ in the HapMap CEU population) and likely tag a single risk locus. Two other SNPs were identified that have Fisher combined p-values near genome-wide significance: rs10519410 on 4q28.3 nearest the PCDH1ggene (combined pvalue $=1.3 \times 10^{-6}$ ) and rs10255878 on 7q21.3 nearest the ACN9gene (combined p-value $=$ $3.6 \times 10^{-6}$ ). Several other SNPs showed a trend towards significance, with combined p-values $<5.0 \times 10^{-4}$ (Supplementary Table 1 ). Though each individual study site in the replication cohort is underpowered to detect statistically significant associations, we confirmed agreement in odds ratio directionality in each study site for all SNPs listed in Supplementary Table 1.

In a related GWAS of erectile dysfunction following radiotherapy, a polygenic risk score representing the sum total number of risk alleles an individual possessed, as a quantitative measure of genetic risk, showed a stronger association signal than any single SNP alone [16]. Here, we see a similar effect using the top 17 SNPs associated with rectal bleeding (excluding rs17630638 and rs7111590, which are in linkage disequilibrium with a SNP already included). After controlling for ethnicity and study site, the polygenic risk score had an odds ratio of $3.0\left(95 \%\right.$ CI 2.4-3.9; p-value $\left.5.4 \times 10^{-18}\right)$ in the discovery cohort and an odds ratio of 1.7 (95\% CI 1.5-1.9; p-value $6.4 \times 10^{-13}$ ) in the replication cohort. Similarly, there was a strong rank-correlation between number of total risk alleles out of these 17 SNPs and rectal bleeding (Sommers' $\mathrm{d}$ p-value $=1.6 \times 10^{-28}$ in the discovery cohort; $5.0 \times 10^{-12}$ in the replication cohort; Table 2). When used to predict the probability of developing rectal bleeding, the polygenic risk score produces an area under the receiver operating characteristic curve (AUC) of 0.892 in the discovery cohort and 0.737 in the replication cohort. It should be noted, the odds ratio and AUC for the discovery cohort is an overly optimistic estimate precisely because this cohort was used for discovery. Furthermore, the per-allele increase in odds of developing rectal bleeding may be non-linear over the set of predictive SNPs, and it will be important to refine this model following validation studies.

Previous studies found SNPs in DNA damage and inflammatory response genes ( ATM LIG4 ERCC2CP2D6*4 MDC1 SOD2 XRCC3and TGFB) to be associated with rectal toxicity. Data was available for 18 of these published SNPs in either some or all of the patients in the current study. Two of the SNPs, rs1800935 in MSH6and rs4803455 in TGFB1 have dominant-model $\mathrm{p}$-values $<0.05$, but these are not significant after correction 
for multiple comparisons (Table 3). All other SNPs available for testing had non-significant p-values.

\section{Discussion}

The rationale for embarking upon the current study is that previous research has been narrowly focused upon specific genes involved in radiation response and DNA repair pathways. We sought to genotype broadly on a genome-wide level and to use the results of the discovery GWAS to focus on a subset of SNPs for validation in replication cohorts. To our knowledge, this is the first genome-wide approach to identify SNPs associated with late rectal toxicity after radiotherapy. Using this approach, we identified a set of candidate SNPs that may be useful in predicting which patients are at risk for development of rectal bleeding following treatment.

Rectal bleeding following radiation is a microangiopathic condition with increased vascular proliferation. The candidate genes located in proximity to some of the significant SNPs in this study may encode products that serve roles in restraining vascular proliferation. The SLC36A4gene that is downstream of the two significant SNPs, rs7120482 and rs17630638, encodes a non-proton coupled amino acid transporter that is required for normal cellular proliferation [17]. Specifically, it modulates the activity of the mammalian target of rapamycin complex 1 (mTORC1) signaling cascade which affects angiogenesis, proliferation and cell survival, among other functions [17]. Interestingly, mTOR signaling has been shown to play a role in radiosensitization in a number of studies across various tumor types, including prostate [18-19]. SNP rs4904509 lies just upstream of FOXN3 which was identified as a novel DNA-damage checkpoint suppressor protein in a yeast genetic screen [20]. This SNP did not show as strong an association signal in the replication cohort, but may warrant further investigation due to its relevant biological mechanism. Finemapping studies will be needed to confirm which genes are affected by the tag SNPs identified here before functional studies can be undertaken.

Our study provides an important replication cohort for the SNPs previously studied in the context of rectal toxicity. None showed significant association after correction for multiple comparisons in this dataset, which had at least $80 \%$ power to detect SNPs whose MAF is greater than $15 \%$ and have an effect size greater than two. A few of the SNPs have very low MAF, and some may be associated but with a small effect size and will need to be investigated in larger cohorts.

There are a few limitations in this study. First, the discovery cohort was treated mainly with brachytherapy whereas the replication cohort was largely treated with EBRT and had lower mean BED (Table 1). While treatment type or dose would not confound SNP association with rectal bleeding, these variables could act as effect modifiers, explaining in part the difference in magnitude of the odds ratios between the discovery and replication cohorts. Despite these treatment differences between cohorts, we were able to identify associated SNPs, suggesting there may be genetic predictors that are non-specific for treatment modality. Another limitation is that different scoring systems were used to assess rectal bleeding, possibly introducing some misclassification bias. However, we believe the scoring systems can be harmonized and that we were able to achieve consistency across cohorts. Finally, while the rectal V100 was not significantly different between cases and controls in the discovery cohort, this data was not available for patients in the replication cohort. It will be important to investigate the possible interaction between rectal V100 and SNPs so that predictive models can include both genetic and non-genetic factors. 
Using a GWAS approach, we identified a locus reaching genome-wide significance for association with rectal bleeding. Additional validation studies are needed to both confirm this finding and to determine whether the other SNPs with marginally significant p-values are in fact associated with late rectal bleeding. Additional studies with larger sample sizes may also uncover additional variants with smaller effect sizes. The current study is an important step in the process of identifying predictors of radiotherapy toxicity that could be used to better tailor therapy to each individual patient.

\section{Supplementary Material}

Refer to Web version on PubMed Central for supplementary material.

\section{Acknowledgments}

This research was supported by grants RSGT-05-200-01-CCE from the American Cancer Society (to BSR), PC074201 from the Department of Defense and 1R01CA134444 from the National Institutes of Health (to BSR and HO), FIS PI10/00164 from the Instituto de Salud Carlos III and FEDER 2007-2013 from Fondo Europeo de Desarrollo Regional (CHUS cohort), and 103.0393.71760001404 through the Alberta Cancer Board Research Initiative Program (CCI cohort).

\section{References}

1. Ferlay J, Shin HR, Bray F, Forman D, Mathers C, Parkin DM. Estimates of worldwide burden of cancer in 2008: GLOBOCAN 2008. Int J Cancer. 2010; 127:2893-2917. [PubMed: 21351269]

2. Grimm P, Billiet I, Bostwick D, et al. Comparative analysis of prostate-specific antigen free survival outcomes for patients with low, intermediate and high risk prostate cancer treatment by radical therapy. Results from the Prostate Cancer Results Study Group. BJU Int. 2012; 109 (Suppl 1):2229. [PubMed: 22239226]

3. Barnett GC, De Meerleer G, Gulliford SL, Sydes MR, Elliott RM, Dearnaley DP. The impact of clinical factors on the development of late radiation toxicity: results from the Medical Research Council RT01 trial (ISRCTN47772397). Clin Oncol (R Coll Radiol). 2011; 23:613-624. [PubMed: 21470834]

4. Bentzen SM, Overgaard J. Patient-to-Patient Variability in the Expression of Radiation-Induced Normal Tissue Injury. Semin Radiat Oncol. 1994; 4:68-80. [PubMed: 10717093]

5. Emami B, Lyman J, Brown A, et al. Tolerance of normal tissue to therapeutic irradiation. Int J Radiat Oncol Biol Phys. 1991; 21:109-122. [PubMed: 2032882]

6. Rosenstein BS. Identification of SNPs associated with susceptibility for development of adverse reactions to radiotherapy. Pharmacogenomics. 2011; 12:267-275. [PubMed: 21332318]

7. Barnett GC, Coles CE, Elliott RM, et al. Independent validation of genes and polymorphisms reported to be associated with radiation toxicity: a prospective analysis study. Lancet Oncol. 2012; 13:65-77. [PubMed: 22169268]

8. Stock RG, Stone NN, Wesson MF, DeWyngaert JK. A modified technique allowing interactive ultrasound-guided three-dimensional transperineal prostate implantation. Int J Radiat Oncol Biol Phys. 1995; 32:219-225. [PubMed: 7721619]

9. Stone NN, Hong S, Lo YC, Howard V, Stock RG. Comparison of intraoperative dosimetric implant representation with postimplant dosimetry in patients receiving prostate brachytherapy. Brachytherapy. 2003; 2:17-25. [PubMed: 15062159]

10. Fachal L, Gomez-Caamano A, Sanchez-Garcia M, et al. TGFbeta1 SNPs and radio-induced toxicity in prostate cancer patients. Radiother Oncol. 2012; 103:206-209. [PubMed: 22385796]

11. Stock RG, Stone NN, Cesaretti JA, Rosenstein BS. Biologically effective dose values for prostate brachytherapy: effects on PSA failure and posttreatment biopsy results. Int J Radiat Oncol Biol Phys. 2006; 64:527-533. [PubMed: 16242258]

12. Purcell S, Neale B, Todd-Brown K, et al. PLINK: a tool set for whole-genome association and population-based linkage analyses. Am J Hum Genet. 2007; 81:559-575. [PubMed: 17701901] 
13. Consortium IH. The International HapMap Project. Nature. 2003; 426:789-796. [PubMed: 14685227]

14. Team RDC. R: A language and environment for statistical computing R Foundation for Statistical Computing. 2.13.1. Vienna, Austria: 2011.

15. Ge, D. MetaP: a program to combine P values. Ge, D., editor. 2009.

16. Kerns SL, Stock R, Stone N, et al. A 2-Stage Genome-Wide Association Study to Identify Single Nucleotide Polymorphisms Associated With Development of Erectile Dysfunction Following Radiation Therapy for Prostate Cancer. International journal of radiation oncology, biology, physics. 2012

17. Heublein S, Kazi S, Ogmundsdottir MH, et al. Proton-assisted amino-acid transporters are conserved regulators of proliferation and amino-acid-dependent mTORC1 activation. Oncogene. 2010; 29:4068-4079. [PubMed: 20498635]

18. Schiewer MJ, Den R, Hoang DT, et al. mTOR is a selective effector of the radiation therapy response in androgen receptor-positive prostate cancer. Endocr Relat Cancer. 2012; 19:1-12. [PubMed: 21903859]

19. Ausborn NL, Le QT, Bradley JD, et al. Molecular profiling to optimize treatment in non-small cell lung cancer: a review of potential molecular targets for radiation therapy by the translational research program of the radiation therapy oncology group. Int J Radiat Oncol Biol Phys. 2012; 83:e453-464. [PubMed: 22520478]

20. Pati D, Keller C, Groudine M, Plon SE. Reconstitution of a MEC1-independent checkpoint in yeast by expression of a novel human fork head cDNA. Mol Cell Biol. 1997; 17:3037-3046. [PubMed: 9154802] 


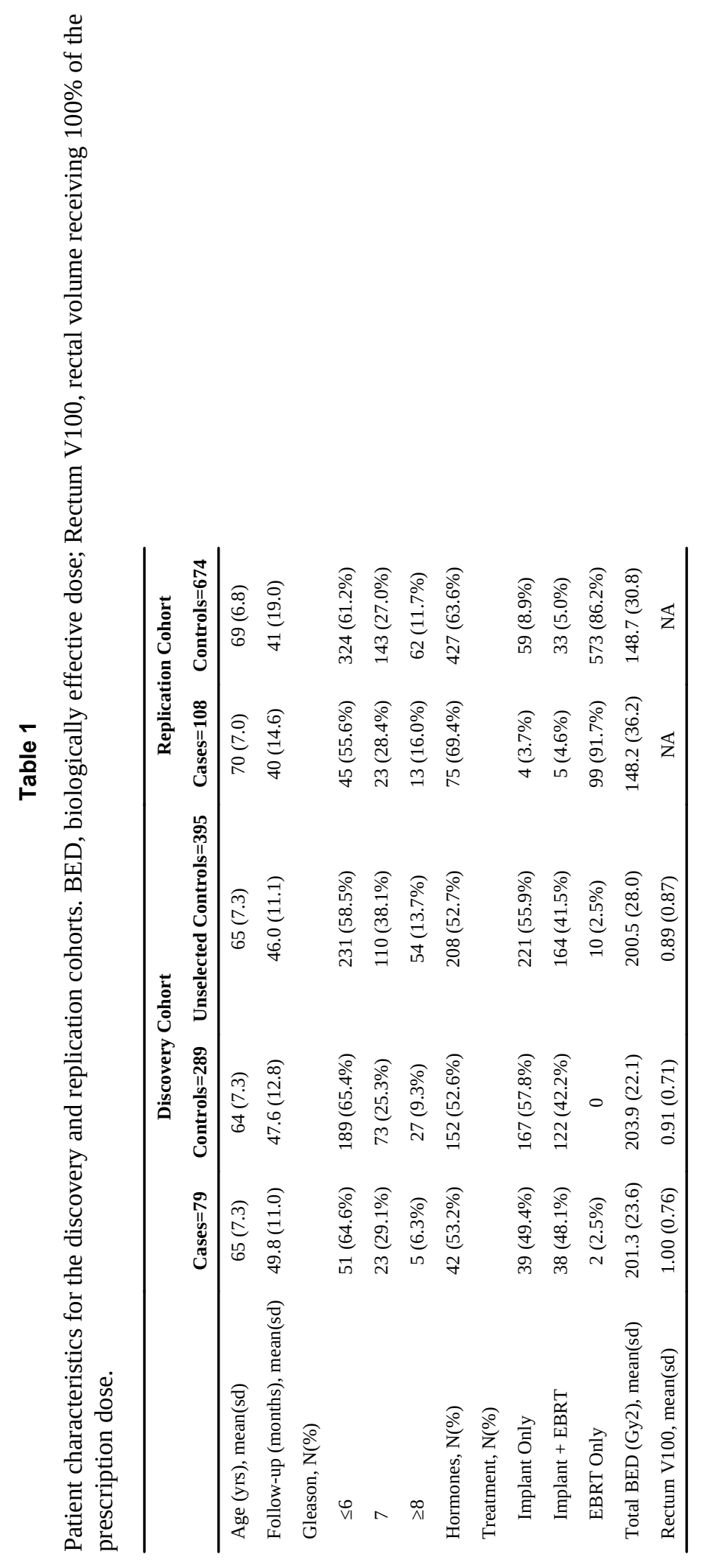


Table 2

Rank-correlation between cumulative number of risk alleles summed for the top 17 SNPs and rectal bleeding case/control status in the discovery and replication cohorts.

\begin{tabular}{|c|c|c|c|c|}
\hline \multirow[b]{2}{*}{ Number of risk alleles } & \multicolumn{2}{|c|}{ Discovery Cohort } & \multicolumn{2}{|c|}{ Replication Cohort } \\
\hline & $\begin{array}{c}\text { Cases } \\
\mathbf{N}=79\end{array}$ & $\begin{array}{c}\text { Controls } \\
N=241\end{array}$ & $\begin{array}{c}\text { Cases } \\
\mathbf{N}=107\end{array}$ & $\begin{array}{c}\text { Controls } \\
N=671\end{array}$ \\
\hline 0 & 0 & 0 & 0 & $1(0.1 \%)$ \\
\hline 0.5 & 0 & 0 & 0 & 0 \\
\hline 1 & 0 & $2(0.7 \%)$ & 0 & $4(0.6 \%)$ \\
\hline 1.5 & 0 & $2(0.7 \%)$ & 0 & $11(1.6 \%)$ \\
\hline 2 & 0 & $12(4.2 \%)$ & 0 & $13(1.9 \%)$ \\
\hline 2.5 & 0 & $25(8.7 \%)$ & 0 & $33(4.9 \%)$ \\
\hline 3 & $1(1.3 \%)$ & $25(8.7 \%)$ & $6(5.6 \%)$ & $48(7.1 \%)$ \\
\hline 3.5 & $1(1.3 \%)$ & $44(15.2 \%)$ & $3(2.8 \%)$ & 48 (7.1\%) \\
\hline 4 & $2(2.5 \%)$ & $42(14.5 \%)$ & $9(8.4 \%)$ & 95 (14.1\%) \\
\hline 4.5 & $2(2.5 \%)$ & 28 (9.7\%) & $9(8.4 \%)$ & $86(12.8 \%)$ \\
\hline 5 & 7 (8.9\%) & $33(11.4 \%)$ & 10 (9.3\%) & 97 (14.4\%) \\
\hline 5.5 & $4(5.1 \%)$ & $32(11.1 \%)$ & $9(8.4 \%)$ & 66 (9.8\%) \\
\hline 6 & $12(15.2 \%)$ & $20(6.9 \%)$ & 15 (14.0\%) & 49 (7.3\%) \\
\hline 6.5 & $5(6.3 \%)$ & $10(3.5 \%)$ & $13(12.1 \%)$ & 40 (5.9\%) \\
\hline 7 & $9(11.4 \%)$ & $3(1.0 \%)$ & $13(12.1 \%)$ & $23(3.4 \%)$ \\
\hline 7.5 & 11 (13.9\%) & $7(2.4 \%)$ & $10(9.3 \%)$ & $16(2.4 \%)$ \\
\hline 8 & $6(7.6 \%)$ & $3(1.0 \%)$ & $6(5.6 \%)$ & $7(1.0 \%)$ \\
\hline 8.5 & $4(5.1 \%)$ & $1(0.3 \%)$ & $1(0.9 \%)$ & $6(0.9 \%)$ \\
\hline 9 & $5(6.3 \%)$ & 0 & $3(2.8 \%)$ & $3(0.4 \%)$ \\
\hline 9.5 & $5(6.3 \%)$ & 0 & 0 & $3(0.4 \%)$ \\
\hline 10 & $2(2.5 \%)$ & 0 & 0 & $1(0.1 \%)$ \\
\hline 10.5 & 2 (2.5\%) & 0 & 0 & 0 \\
\hline 11 & $1(1.3 \%)$ & 0 & 0 & 0 \\
\hline
\end{tabular}




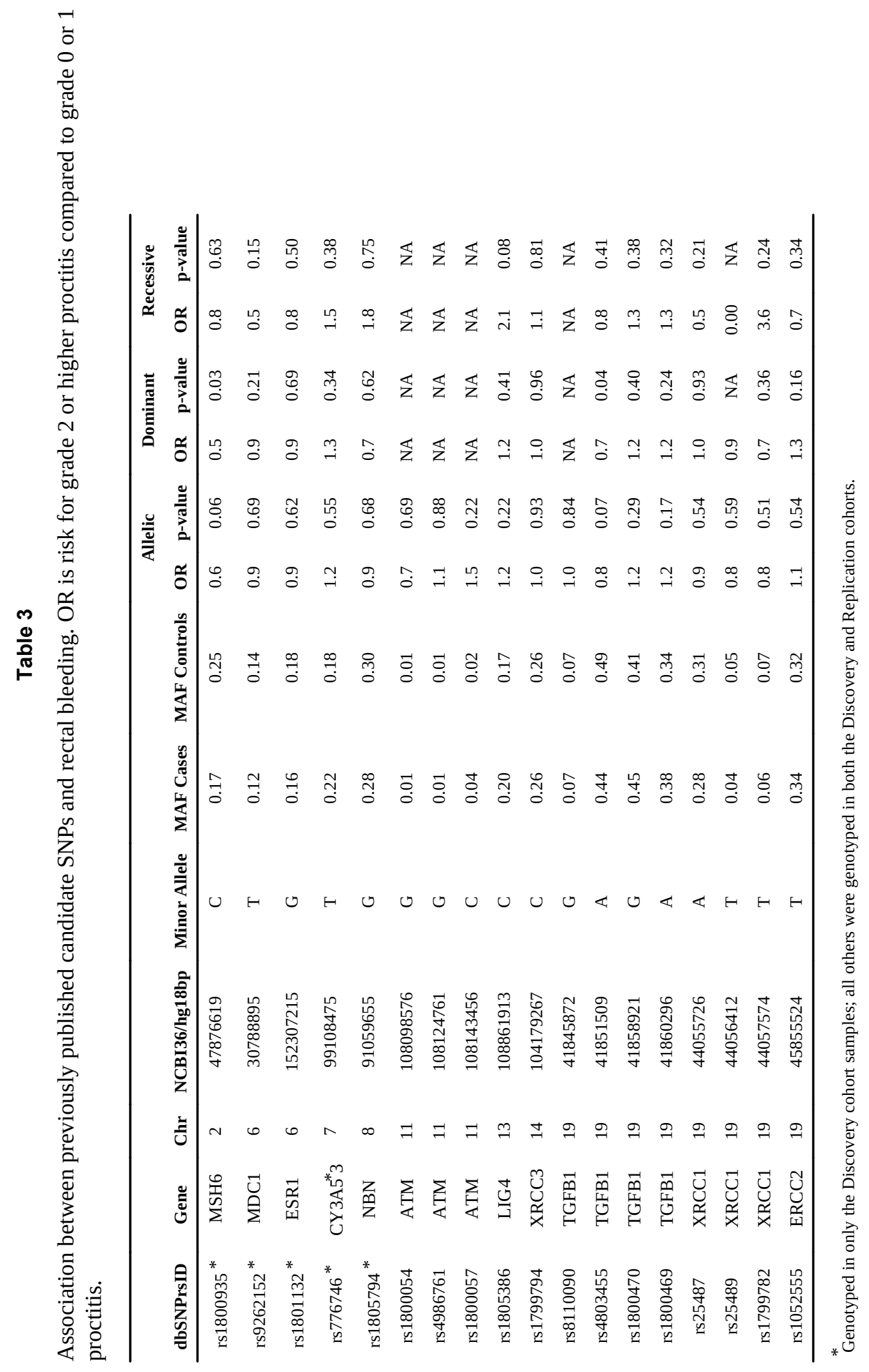

Radiother OncolAuthor manuscript; available in PMC 2014 June 01. 